\title{
Platform Presentations
}

1.

\section{Evaluation of EMG Expert Systems}

A. FUGLSANG-FREDERIKSEN, B. JOHNSEN, S. VINGTOFT, J. RØNAGER, P. FAWCETT, I. SCHOFIELD, R. LIGUORI, W. NIX, G. OTTE, G. SIEBEN, M. VELOSO and A. VILA

(Copenhagen, Denmark; Newcastle upon Tyne, United

Kingdom; Bologna; Mainz; Gent; Lisbon; Grenoble)

In the development of EMG expert systems evaluation play a very important role as illustrated in the following.

KANDID is a Knowledge-based Assistant for Neuromuscular Disorder Diagnosing implemented on a PC in Prolog. This ruled-based EMG expert system is an assistant to the physician using an iterative plan-test-diagnose cycle. It covers the full range of neuromuscular disorders. The expert system performs data reduction and categorical uncertainty handling of test results into pathophysiological states and categorical likelihood grouping of EMG disorder hypotheses.

A prospective evaluation of KANDID at seven European laboratories indicated great interlaboratory differences in control material, techniques and strategies. KANDID performed best where the "Buchthal school approach" was used. We learned from this study that: 1) better formalized evaluation methodologies have to be developed; 2 ) there should be consensus on diagnoses of cases tested; 3 ) it should be possible to take into account differences in techniques and strategies; 4) an evaluation is very time consuming and an automatic evaluation tool is necessary.

To overcome these problems ESTEEM, an AIM-project within EEC has been initiated. The purpose is to construct an EMG communication platform containing a database of reference patient cases with consensus on diagnoses, expert systems, an evaluation tool and interfaces to EMG equipment. This platform can be used for testing of expert systems and for interlaboratory communications.

Patient cases are collected in a common European database and a procedure to seek consensus on diagnoses and intermediate pathophysiological states of anatomical structures is carried out among nine neurophysiologists. Differences in the diagnostic process are revealed by an analysis of used examination techniques, planning strategies, normal material and diagnostic criteria. The database will serve as a reference for the evaluation of the diagnostic performance of future expert systems.

A general structure for the representation of EMG data has been made. This structure allows for the storage of data from all examination techniques used in routine at seven European EMG laboratories including diagnoses and intermediate pathophysiological states of anatomical structures.

The platform EMG including the data base of patient cases will make it possible to obtain the necessary information for the development of second generation EMG expert systems and to make a proper evaluation.
2.

\section{A Neural Network Classifier in MUAP Analysis}

\section{L.T. MIDDLETON, C.S. PATTICHIS, R. CONSTANTINOU and C.N. SCHIZAS (Nicosia, Cyprus)}

In the past years several computer-aided quantitative motor unit action potential (MUAP) techniques were reported. It is now possible to add to these techniques the capability of automated medical diagnosis so that all data can be processed in an integrated environment. In this study, the parametric pattern recognition (PPR) algorithm that facilitates automatic MUAP feature extraction, and Artificial Neural Network (ANN) models are combined for providing an integrated system for the diagnosis of neuromuscular disorders.

Learning in ANN models is accomplished through systematic training with known data. Two paradigms of learning for training ANN models were investigated, supervised and unsupervised. For supervised learning the back propagation algorithm and for unsupervised learning the Kohonen's self-organizing feature maps algorithm were used. In supervised learning the training data is supplied at the input and the generated output is compared with the desired output; the error difference is used for modifying the ANN connections until learning is accomplished. In unsupervised learning, data is presented sequentially at the input without specifying the desired output. These models are capable of self organization; thus, topologically close output nodes become sensitive to physically similar inputs. Diagnostic yield for models trained with both procedures was similar and of the order of $80 \%$. However, back propagation models required considerably more computational effort compared to Kohonen's self-organizing feature map models.

Furthermore, in order to enhance the diagnostic performance of neural network models, a hybrid system was developed that employs both back propagation and self-organizing feature map models.

\section{3.}

From Expert Systems to Case-Based Reasoning Systems: Neuromyosis, An Example of the Artificial Intelligence Adventure in EMG

\section{ANNICK VILA (Grenoble, France)}

During diagnosis reasoning, the main asset of a senior doctor is his experience. This experience finds expression in the know!edge of a set of representative cases in his field. In the domain of EMG diagnosis, novice EMG'ers fail through either excess or insufficiency. In the first instance, they suggest test strategies which are cumbersome, painful for the patient and expensive, for fear of missing tests and inconclusive results. The reasoning and the resultant examination are thus excessive. Conversely, in 
the second case, they tend to suggest an insufficient EMG examination without evoking, for example, multiple disorders and the necessary tests for their confirmation, thus producing an inaccurate diagnosis. To decrease these problems, different knowledge based systems (KBS) have been developed to provide an aid to EMG diagnosis: Expert systems modelise the expertise of one individual human expert, KBS integrate some consensual knowledge of a community. But this knowledge is static. The interest of a case-based reasoning system (CBRS) is to add to a KBS the dynamic aspect of experience, taking into account the variability of the clinical situation and the necessary adaptable planning strategy. Actually to establish a diagnosis a KBS proposes one strategy according to a fixed reasoning process considered to be the most accurate and representative; to modelise the experience, CBRS propose different strategies for each diagnosis hypothesis according to the clinical and technical situation. So we now propose the construction of a knowledge based system which integrates a case based reasoning module into the NEUROMYOSYS system, allowing the novice EMG'er to be helped in his choice of test strategies and diagnosis reasoning.

\section{4.}

\section{EMG Cases As Seen by the Expert System Munin, by EMG} Experts and by the Audience

\section{STEEN ANDREASSEN, BJØORN FALCK and KRISTIAN G. OLESEN (Aålborg, Denmark; Uppsala, Sweden)}

The diagnostic function of a prototype of the MUNIN expert system for EMG will be described. The prototype contains a reasonably detailed description of the most important groups of generalized muscle and nerve disorders, but the prototype is limited to a small part of the neuromuscular anatomy. The implemented "Microhuman" anatomy has 6 muscles and 8 nerves, and a correspondingly limited number of local nerve lesions. The prototype can handle results from most of the commonly used EMG and nerve conduction techniques. The prototype can be used both for "diagnostic" and for "causal" reasoning. In the former type of reasoning the prototype is used to diagnose, i.e., the systems probabilistic inference engine is used to reason from test results through different aspects of neuromuscular pathophysiology to disorders. In the latter form of reasoning, causal reasoning, the inference engine can be used to provide descriptions of individual disorders: the system reasons from disorders through pathophysiology to expected test results.

Following this description of the prototype, the systems function will be illustrated by a number of cases, which two EMG'ers, Jasper Daube and Erik Stålberg, have agreed to provide. They will comment on MUNIN's possible applications as a diagnostic aid and as a teaching tool. Diagnostic suggestions will be elicited from MUNIN, from the EMG'ers that provided the cases and from the audience.

\section{5.}

\section{Sprouting, Regeneration and Growth Factors}

\section{JACK DIAMOND (Hamilton, Ontario)}

Denervated target tissues become reinnervated only if there is regeneration of damaged nerve fibres, and/or collateral sprouting of undamaged ones. To learn some of the "ground rules" that govern these two growth processes, we study the reinnervation of denervated skin. An obvious difference between them is the way they are evoked, namely by axonal damage for regeneration, and by the provision of nerve-depleted target tissue for collateral sprouting. We have recently characterized another distinguishing and quite unexpected feature. AntiNGF treatment totally blocks the collateral sprouting of intact nociceptive and sympathetic fibres into denervated skin, but is without effect on the regeneration of the same nerves after damage. This holds even when the regenerating axons are extending into the denervated skin surrounding their original field of innervation, growing along the same pathways used by the NGF-dependent collateral sprouting that would have occurred had the nerves been spared. In accord with these observations, we now find that in collaterally sprouting, but not regenerating, DRG neurons, the expression of low and high affinity NGF receptors increases markedly; NGF is known to cause such increases. The vigour of axonal regeneration appears to correlate with the extent of the morphological and biochemical changes evoked in the neuron by axotomy, known collectively as the cell body reaction (CBR). For example, the CBR and the regenerative response both increase with multiple axotomies, and both are reduced by making the axotomy further from the cell body. Recently we used 6 hydroxydopamine (6-OHDA) to destroy only the terminal and paraterminal regions of the sympathetic axons; the morphological signs of a CBR are then nondetectable. We find the subsequent development of a functional sympathetic field to have two phases. The first occurs independently of NGF, and is limited in extent; it appears to correspond with regeneration. The ensuing phase is virtually unlimited while denervated skin is available, and is wholly NGF-dependent; this growth correlates with collateral sprouting. We then tested "repeated axotomies" by administering multiple injections of 6-OHDA over a number of days; this significantly increased the extend of the initial (NGF-independent) phase of the recovery.

Generalized, it seems that collateral sprouting, which occurs independently of a CBR (none has been reported), is initiated and sustained entirely by target-derived neurotrophins, while regeneration occurs independently of these, and instead is triggered by a signal generated by axotomy, and driven by a central program which is intimately associated with a CBR. Somewhat paradoxically however, collateral sprouting can be influenced by impulses in the neuron, while regeneration cannot.

\section{6.}

\section{Motor Unit Number Estimation: A Review}

\section{JASPER R. DAUBE (Rochester, U.S.A.)}

The number of motor units in a muscle or group of muscles is a critically important measure of the severity and progression of a neurogenic disorder of either peripheral nerve or anterior horn cells. The number of motor units in a muscle is ordinarily evaluated by assessment of recruitment on needle electromyography, a subjective method, that provides only coarse estimates without numerical quantitation. 
Methods that provide quantitative measures of the number of motor units have been devised over the past twenty years, but none have received wide acceptance because of technical limitations and difficulty with application. These have included measurement of compound muscle action potential increments near threshold, measurement of sizes of F-waves, measurement of spike triggered averages from motor unit potentials recorded on needle electromyography and statistical estimates of compound muscle action potential variation. Modifications of a number of these methods have been devised to improve on their accuracy and reproducibility.

Each of them relies on the basic principle of estimating the average size of individual, surface recorded, motor unit potentials by one of the potential is then divided by this average motor unit potential size to estimate the number of motor units. These methods have inherent assumptions in common with each other. Most important is the assumption that the true average size can be measured with a limited sampling of the motor units. This may not be correct, particularly in the presence of disease. In addition, for each method there may be a selection bias in the size of motor units selected for measurements.

The original method of McComas measures the size of increments near threshold to determine an average motor unit size. This method has been extended with means of automation, and by modifications of the methods of stimulation to widen the range of axons selected. The stimulation has been applied at different voltage levels, at different points along the nerve, and with difference recording electrode arrays.

The F-wave method assumes that some of the F-waves are the potentials of single motor units. While this is true, the many combinations in which multiple $\mathrm{F}$-waves can summate results in the number of different $F$-wave configurations from a given number of F-waves, expanding factorially with the number of contributing $\mathrm{F}$-waves.

Spike triggered averaging has used both voluntary activation of motor unit potentials or stimulation of nerve terminals in the muscle. The corresponding surface recordings to these potentials of single motor units isolated with a needle electrode, are then averaged to obtain a surface representation of the motor unit potential. While the method is effective, it can be more time-consuming than is acceptable for routine clinical testing.

Statistical methods rely on the inherent instability of the firing of an axon near threshold resulting in spontaneous, all-ornone variations in the size of the compound muscle action potential. Using the assumption of a Poison distribution of the sizes of the potentials, it is possible to calculate mean potential size and from that, motor unit estimate.

It has been most gratifying for the continued development of motor unit number estimates, that each of these methods gives values in the same range. For the most commonly tested thenar and extensor digitorum brevis muscles the normal ranges are 100-500, and 50-300 respectively for individual normal subject. Further development and automation of these techniques will provide valuable new measurements of the stage of a neurogenic disease, and provide better methods for following the course of the diseases.
7.

\section{London-Boston Systems for Deriving Motor Unit Estimates}

W.F. BROWN, T. DOHERTY and D. STASHUK (Boston, U.S.A.; London, Ontario; Waterloo, Ontario)

One of the most serious detractions in the original manual incremental stimulation technique for estimating the number of motor units in various muscles was the fact that the thresholds of motor axons so commonly overlap, especially at stimulus intensities exceeding all but the lowest threshold motor axons at any one stimulus site. The resulting problems presented by "alteration" plagued the original method and frustrated application of the technique in many laboratories. From its inception, however, the method was recognized to be much easier to carry out in peripheral neuropathies and motor neuron diseases where alternation was much less troublesome because there were fewer motor axons and the thresholds of "the few" were often sufficiently widely separated from one another to allow for reliable estimates to be carried out.

Recently, several groups have introduced different techniques for circumventing the alternation problem, often with the aid of computer programs designed to identify alternation or take advantage of alternation when present. Our approach has been to avoid alternation entirely by deriving a representative sample of single, surface-detected motor unit action potentials (S-MUAPS) using one of three techniques. These include threshold electrical stimulation at multiple sites along the course of the nerve (MPS) collecting only the "all" responses of the first or perhaps second motor axon at each stimulus site. Alternatively, we have developed computer assisted manual and fully automated techniques for collecting the sample of single SMUAPS from among the F-responses evoked by a train of several hundred stimuli delivered to the motor nerve. The third option has been to combine isometric contraction to recruit motor units and spike-triggered averaging to select the requisite sample of S-MUAPS.

Each method carries with it advantages and disadvantages, and some methods are more applicable to certain muscle groups than others. Nonetheless, despite the widely differing possible biases in each of these techniques, estimates of motor unit numbers using all three methods have been in close agreement with one another and with estimates derived using other methods.

\section{8.}

\section{Volume Conductor Modelling of the Surface EMG: Principles and Results}

D.F. STEGEMAN, A.J. VERSLUIJS, J. MOLEMAN and $\mathrm{K}$. ROELEVELD (Nijmegen, The Netherlands)

Motor unit counting techniques depend on the interpretation of surface EMG parameters. It is usually implicitly assumed that the EMG signal is equally representative for all regions within a muscle. With electrophysiological modelling, the relative contributions from different parts of a muscle can be predicted. However, a volume conductor model can only be used for the prediction of surface EMG parameters when some essential 
elements are included: (i) a finite volume conductor representing the limb, (ii) finite instead of infinite length of muscle fibres and (iii) inhomogeneities representing the deviating electric conductivity of the subcutaneous fat layer. A model fulfilling the above demands was developed. ' Within that model a muscle is postulated which consists of a number of motor units at different locations. EMG amplitude and waveform characteristics are evaluated as a function of cross-sectional coordinates of the muscle. The influence of different lead-off schemes and different interelectrode distances is estimated as well. Most of the results confirm, but also quantify, intuitive expectations, such as a prominent depth dependence of the EMG amplitude contribution and a non-uniform waveform contribution from different parts of the muscle. With decreasing inter-electrode distances and increasing "order" of lead-off schemes (monopolar, bipolar or even higher order) the EMG signal becomes more "superficial" and less representative for the muscle as a whole.

'Gootzen, Stegeman, van Oosterom. Electroenc Clin Neurophysiol 1991: 81: $152-162$.

\section{9.}

\section{Clinical Experience with the McMaster Motor Unit Estimating System}

\section{A.J. McCOMAS (Hamilton, Ontario)}

We now have three years' experience with the McMaster automated motor unit estimation (AMUE) system in the EMG clinic and have compared the results with data from 238 muscles of healthy control subjects below the age of $60 \mathrm{yr}$. The patients were unselected and have included those with suspected entrapment neuropathies and radiculopathies, as well as those with generalized denervating disorders. Usually AMUE was performed prior to motor conduction velocity measurements, since the same electrodes could be employed for both procedures. The thenar and hypothenar muscle groups, the extensor digitorum brevis and the biceps brachii were more frequently examined than the vastus medialis, tibialis anterior and plantar group. AMUE was repeated only when borderline or reduced values were obtained in the initial examination. In approximately $10 \%$ of cases difficulties were encountered of one kind or another (background muscle activity, mains interference, patient compliance) but in the remainder useful results were obtained. Illustrative cases will be presented from the more than 400 muscles investigated in the patient population.

Research supported by: the Muscular Dystrophy Association of Canada and the De Groote Foundation.

10.

\section{Reference Values in SFEMG}

ERIK STÅLBERG and JOZE TRONTELJ (Uppsala, Sweden; Ljubljana, Slovenia)

Fibre density - Fibre density (FD) is a sensitive parameter to detect abnormal organization of muscle fibres in a motor unit. A multicenter study (Gilchrist et al. 1992) has shown that the method is sufficiently standardized to allow comparison between different laboratories. The FD value varies between muscles and increases with age to different degrees for different muscles. Published values must be consulted in the individual case.

Jitter, voluntary activity - The neuromuscular jitter is the parameter that reflects neuromuscular transmission in individual motor end-plates. In the normal muscle there is a considerable range of jitter values, and this also applies to motor end-plates belonging to a single motor unit.

The physiological degree of irregularity, which occurs at steady contraction, does not influence the jitter (at least for MIPI less than $4 \mathrm{~ms}$ ), but at very irregular innervation rates the jitter sometimes increases slightly. A decrease of the intramuscular temperature below $35^{\circ} \mathrm{C}$ increases the jitter in normal muscle by $1-3 \mu \mathrm{s} /{ }^{\circ} \mathrm{C}$. The normal individual values range between 5 and 35-60 $\mu$ s (MCD) for different muscles, with some exceptional isolated values above this limit, not more than one in any tested subject. The upper limit for the mean value of 20 recordings in one muscle is of the order of 30-50 $\mu$ s, depending on the muscle. Most muscles show changes after the age of 50. A muscle is considered abnormal if 2 of more individual data are above given limits or if the mean value is outside the limits.

A multicenter study (Gilchrist et al. 1992) ${ }^{1}$ was undertaken to establish reference values.

Jitter, axonal stimulation - With electrical stimulation the jitter is measured between the stimulus and a single muscle fibre action potential, hence from only one motor end-plate at a time. The values obtained are therefore lower, theoretically on the average by a factor of $1 / \sqrt{ } 2$, i.e., $71 \%$ of the jitter values obtained in voluntarily activated pairs. For muscles in which specific reference values are missing, it is safe to use the values given for voluntary activation multiplied by a conversion factor of 0.8 .

'Gilchrist. J.M. et al. Single Fibre EMG reference values: A collaborative effort. Muscle and Nerve 1992; 15: 151-161.

\section{1.}

\section{Stimulation SFEMG: Validation of the Method and Uses}

JOZE TRONTELJ and ERIK STÅLBERG (Ljubljana, Slovenia; Uppsala, Sweden)

Stimulation SFEMG, although being around since 1966, has relatively recently become an established method for jitter measurement. ${ }^{1.2}$ It can conveniently replace the original method in voluntarily activated muscle, particularly when the patient cannot co-operate for a voluntary SFEMG study, e.g., because of young age or psychological disturbance or severe weakness, whether due to a central or a peripheral cause. It effectively removes the interdischarge-interval dependent jitter. Besides, the stimulation method is faster, as there is no need to search for muscle fibre pairs (necessary for time reference in voluntary jitter measurement). Only one neuromuscular junction (NMJ) is studied at a time, thus quantitative studies are easier and more exact.

During the stage of development, the following questions were a major concern and had to be answered before the method could be validated: 1) Is there a significant amount of jitter generated at the stimulated site on the axon? 2) Are there reliable precautions to avoid the false large jitter and blocking due to 
inadvertent threshold stimulation? 3) Can one identify direct muscle stimulation in order to avoid false normal results?

Among the uses of the method are studies of transmission at single NMJs at wide ranges of firing frequencies and during various, well defined patterns of rest and activity. Thus it is possible to quantitate the contribution of such processes as inter-and post-tetanic facilitation and exhaustion, and, with some degree of reliability, distinguish between the "postsynaptic type" of disturbed transmission such as in myasthenia and the "presynaptic type" such as in LEMS.

The user however has to be continuously aware of certain pitfalls, the most serious of which is inadequate stimulus strength.

'Stålberg, E., Trontelj, J.V., and Mihelin, M. Electrical micro-stimulation with single fibre EMG: A useful method to study the physiology of motor unit. J Clin Neurophysiol 1992; 9: 105-119.

${ }^{2}$ Trontelj, J.V. and Stålberg, E. Jitter measurements by axonal stimulation. Guidelines and technical notes. Electroencephalogr Clin Neurophysiol, EMG and Motor Control 1992; 85: 30-37.

12.

\section{Single Fibre EMG (SFEMG) Using Voluntary Activation}

\section{DONALD B. SANDERS (Durham, U.S.A.)}

Measurement of jitter by SFEMG is the most sensitive in vivo technique to assess neuromuscular transmission. Thus, jitter measurements provide the most sensitive demonstration of the physiologic abnormality in myasthenia gravis (MG). Most patients can cooperate well enough to obtain studies during voluntary activation, including children older than 8 years. I prefer using voluntary activation in virtually all SFEMG studies performed for diagnostic purposes, finding it faster and less subject to artifacts than stimulated jitter studies. It may be difficult to obtain good recordings with voluntary activation in limb muscles in patients with tremor; in these patients, I can usually get the necessary information by studying a facial muscle.

SFEMG demonstrates abnormal jitter in at least one muscle in virtually all $(99 \%)$ patients with MG. To obtain the maximum diagnostic sensitivity it may be necessary to examine several muscles, including ones that are more involved clinically. The muscles to be tested should be selected based on the distribution of weakness in the patient. If there is weakness in limb or oropharyngeal muscles, I usually test the EDC first. In MG patients with weakness limited to the ocular muscles, the EDC may be normal and I usually examine the frontalis muscle first in these patients. In occasional patients with ocular myasthenia the frontalis is normal but jitter is increased in the orbicularis oculi. Thus, I do not consider a study to be normal in patients with suspected ocular MG unless the frontalis and orbicularis oculi have been examined.

Abnormal jitter is also seen in diseases of nerve and muscle; these diseases must be excluded by other electrophysiologic and clinical examinations before diagnosing MG. In most patients with MG, jitter is greater in facial than in limb muscles; the converse is true in peripheral nerve disease and in most myopathies. An exception is oculocraniosomatic myopathy, in which jitter is frequently increased only in facial muscles. If neuronal or myopathic disease is present, increased jitter does not indicate that MG is also present. However, if jitter is normal in a muscle with definite weakness, the weakness is not due to MG. When abnormal neuromuscular transmission has been demonstrated by repetitive nerve stimulation, the finding of abnormal jitter does not add to the diagnosis, although it may be useful in providing baseline values for comparison with the results of subsequent studies. SFEMG is most valuable clinically in the patient with suspected MG in whom other tests of neuromuscular transmission and anti-acetylcholine receptor antibody titers are normal. Serial measurements of jitter can be useful in following the course of disease and in assessing the effect of treatment, but the results from these studies must always be interpreted considering the overall clinical picture.

13.

\section{Macro EMG in the Diagnosis of Neuromuscular Disorders}

\section{P.R.W. FAWCETT (Newcastle upon Tyne, U.K.)}

Macro EMG recordings are made from the terminal $15 \mathrm{~mm}$ portion of exposed cannula of a modified Single Fibre electrode, which because of its relatively large recording surface sees activity from the whole motor unit, providing a measure of its overall (electrical) size. Simultaneously recorded SFEMG signals are used to trigger on individual motor units and determine the fibre density (FD) and jitter. Recordings are obtained during slight voluntary contraction at 20 different sites in the muscle; the amplitude and area of each Macro motor unit potential (Macro MUP), number of negative peaks and the median value and range for the 20 recordings are determined.

In the muscular dystrophies (MD) and other myopathies Macro MUP amplitudes are generally low or normal and the FD values are normal or only moderately increased, reflecting local reorganization within the motor unit and slight reductions in motor unit size. The number of peaks is increased and they tend to show temporal dispersion. Occasionally in some patients with Becker and facioscapulohumeral MD, Macro MUP amplitudes are significantly increased, but the corresponding FD is normal, suggesting muscle fibre hypertrophy rather than an increase in the number of muscle fibres in the motor unit.

By contrast in neurogenic conditions, parallel increases in FD and Macro MUP amplitudes are seen, reflecting the degree of compensatory reinnervation locally and throughout the motor unit. These changes are most marked in chronic neurogenic states such as spinal muscular atrophy and old poliomyelitis with Macro MUPs of 20-30 times the normal amplitude. In motor neurone disease (MND), increased Macro MUP amplitudes are seen, but the degree of the increase depends upon the rate of evolution of the disease process in the muscle under study.

The combination of FD and Macro MUP amplitude measurement has proved to be a reliable means of differentiating between myopathic and neurogenic disorders.

\section{4.}

\section{Concentric-Macro EMG in Neuropathic and Myopathic Disorders}

\section{J.F. JABRE (Boston, U.S.A.)}

Goal: We have set out to study the Local and Global motor unit changes in Neuropathic and Myopathic diseases of the 
motor unit. A special needle built by covering the cannula of a conventional Concentric electrode with Teflon ${ }^{\mathrm{TM}}$ insulation leaving bare a $15 \mathrm{~mm}$ section (the ConMac electrode) was used.

Background: Conventional Concentric electrode studies may not reflect the overall changes in the territory of a motor unit as they sample a relatively small number (15-20) of muscle fibres in the unit. Simultaneous recording of the Macro potential (in this case referred to as the ConMac potential) from the cannula of the Concentric electrode enables the study of a small and a large section of the motor unit at the same time.

Methods: A total of 14 patients were studied. They consisted of 7 patients with various Neurogenic conditions: Polio (2); Motor Neuron Disease (2); Peripheral Neuropathy (2); and Syringomyelia (1); and 7 patients with various Myopathic conditions: Steroid Myopathy (1); Congenital Myopathy (1); Disuse Atrophy (3); and Polymyositis (2).

Results: On average, the Concentric Motor Unit Action Potential (MUAP) of the Neurogenic units increased by 3.5 fold (when compared to normals) while the Concentric MUAP of Myopathic unit was roughly $2 / 3$ normal. By contrast, the ConMac MUAP of the same Neurogenic MUAPs was 7 times larger than normal while those of the Myopathic MUAPs were $2 / 3$ normal (same as the Concentric). These findings could be explained by the "biasing" which occurs with the Concentric electrode in diseases involving reinnervation as the number of muscle fibres in its immediate vicinity reaches a critical threshold. By its sheer size, the Macro surface prevents biasing and thus reflects the true size or "Fibre Population" of the motor unit. The same is not true for Myopathic units since biasing does not occur when there is a decrease in the number of muscle fibres in the unit. The difference between Neurogenic and Myopathic ConMac MUAPs (from 2/3 decrease to 7 fold increase) is roughly twice that of the concentric MUAP.

Conclusion: Concentric-Macro electrode studies give a good appreciation of the changes in the Motor Unit size and reflect more accurately the "Fibre Population" of a motor unit in Neurogenic and Myopathic disorders than do Concentric studies alone.

15.

\section{What Can EMG Learn from EEG?}

\section{ADRIAN R.M. UPTON, (Hamilton, Ontario)}

Sherrington (1906)' emphasized the integrative action of the nervous system. Now that there are so many sophisticated tests available to the clinician it is easy to focus on one area of technique at the expense of others and the physical separation of EMG and EEG laboratories may not be an optimal use of resources.

This conference is devoted to the recent developments in computerized and quantitative electromyographic techniques but the value of EMG studies can be supplemented or multiplied by concurrent use of EEG technology.

For example, the introduction of magnetic stimulation ${ }^{2}$ has provided a method of assessing motor pathways from cortex to muscle fibre. Evoked potentials can demonstrate function of afferent pathways that cannot be measured adequately by peripheral or electromyographic techniques. More recent methods of recording autonomic potentials ${ }^{3}$ has allowed assessment of sympathetic pathways.
This paper will discuss the methodology and clinical applications of these techniques which combine peripheral and central pathways or EEG, EMG and even ECG techniques.

Some afferent pathways from visceral structures such as the bladder, the bowel, the oesophagus or the heart can only be measured by central or "EEG" techniques. As a supplement to the results of EMG studies evoked potentials from the back, neck or head can be valuable in assessment of dermatomal, peripheral nerve and autonomic pathways. Evoked potentials may provide the best method of assessment of the trigeminal, ${ }^{4}$ lateral cutaneous femoral nerve and pudendal nerve responses in males and females. ${ }^{5}$

Segmental and dermatomal evoked potentials can evaluate the effects of peripheral nerve, root, spinal or central damage. ${ }^{6}$ These techniques can be used in the unresponsive or unconscious patient or in the neonate and infant. Brachial plexus and spinal potentials can help to evaluate proximal nerve damage in all age groups. ${ }^{7}$

Delays in visual, auditory or somatosensory pathways may be associated with a number of neuromuscular disorders (e.g., spinocerebellar degeneration) and delays of demyelination may explain complaints of fatigue or fluctuating weakness and numbness.

Although autonomic function can be measured by technically demanding microneuronography ${ }^{8}$ which can be regarded as an EMG technique, the results do not provide wide enough sampling or a practical method of clinical testing. ${ }^{9}$ Recent developments in recording vagal and oesophageal evoked potentials and $\mathrm{R}-\mathrm{R}$ interval changes in the electrocardiogram (EKG or ECG) ${ }^{10}$ demonstrate the clinical value of combining (or integrating) multiple techniques (EEG, EMG, EKG) in a single practical clinical testing procedure.

Back averaging of the EEG can be used to assess a myoclonic discharge on EMG. The EMG discharge is used to generate a triggering pulse that drives the EEG averages and this can measure corticospinal (shorter latencies of 6 to $22 \mathrm{msec}$ ) or less direct pathways (e.g., 50 to $80 \mathrm{msec}$ )."

The interdependence of peripheral and central pathways is illustrated by trophic effects and transynaptic degeneration in motor and sensory pathways ${ }^{12-14}$ and it is probable that increasing use of combined EEG and EMG techniques will demonstrate widespread effects of apparently localized lesions including central effects of peripheral lesions and peripheral effects of central disturbances.

In the future the application of limited stimulus or single stimulus averaging techniques will make it possible to make practical use of cortical potentials (e.g., C.N.V., post imperative negative variation) and late responses (e.g., $V_{1}, V_{2}$ ) in the assessment of motor disorders. There is evidence that cortical excitability is depressed in patients with A.L.S. ${ }^{15}$ as measured by a combination of transcranial magnetic stimulation and muscle responses. All of these results help to emphasize the importance of integration of EMG and EEG laboratories and techniques.

'Sherrington, C.S. The integrative action of the nervous system. Charles Scribner \& Sons, New York, 1906.

${ }^{2}$ Merton, P.A. and Morton, H.B. Stimulation of the cerebral cortex in the intact human subject. Nature 1980;285: 227.

'Upton, A.R.M., Tougas, G., Talalla, A., White, A., Hudoba, P., Fitzpatrick, D. Clarke, B. and Hunt, R. Neurophysiological effects of left vagal stimulation in man. PACE 1991: 14: 70-76. 
${ }^{4}$ Leandri, M., Parodi, C. and Favale, E. Normative data on scalp responses evoked by infraorbital nerve stimulation. Electroenceph Clin Neurophysiol 1988; 71: 415.

'Haldeman, S., Bradley, W.E. and Bhatia, N. Evoked potentials from the pudendal nerve. J Urol 1982; 128: 974.

'American Electroencephalographic Society, Guidelines from Clinical Evoked Potentials Studies. J Clin Neurophysiol 1984; 1: 3.

'Yiannikas, C., Shahani, B.T. and Young, R.R. The investigation of traumatic lesions of the brachial plexus by electromyography and short latency somatosensory potentials evoked by stimulation of multiple peripheral nerves. $J$ Neurol Neurosurg Psychiatry 1983; 46: 1014.

${ }^{8}$ Faguis, J. and Wallin, B.G. Sympathetic reflex latencies and conduction velocities in normal man. J Neurol Sci 1980; 47: 433.

'Upton, A.R.M. and White, A.M. Autonomic stimulation. PACE 1991; 14: 50-69

${ }^{10}$ Kamath, M.V., Upton, A.R.M., Talalla, A. and Fallen, E.L. Neurocardiac responses to vagoafferent electrostimulation in humans. PACE 1992; 15: 1581-1587.

"Shibasaki, H., Neshige, R. and Hashiba, Y. Cortical excitability after myoclonus: jerk-locked somatosensory evoked potentials. Neurology 1985; 35: 36.

12McComas, A.J., Sica, R.E.P., Upton, A.R.M. and Currie, S. Motorneuron dysfunction in patients with hemiplegic atrophy. Nature, New Biology 1971; 23: $21-23$.

${ }^{13}$ McComas, A.J. and Upton, A.R.M. Transneuronal degeneration as basic mechanism of muscular atrophy of central origin. Nature, New Biology 1972: 238: 285-286

${ }^{14}$ Aisen, M.L., Brown, W. and Rubin, M. Electrophysiologic changes in lumbar spinal cord after cervical cord injury. Neurology 1992; 42: 623-626.

1.5isen, A., Pant, B. and Stewart, H. Cortical excitability in amyotrophic lateral sclerosis: a clue to pathogenesis. Can J Neurol Sci 1993: 20: 11-16.

16.

\section{Fatigue in Neuromuscular Disease}

\section{ROBERT G. MILLER (San Francisco, U.S.A.)}

Fatigue is one of the most common problems in clinical medicine. New methods are available which permit quantitation of different sites of failure in the fatigue process. Central fatigue, impaired excitation-contraction coupling, and changes in muscle energy metabolism can all be measured in a quantitative fashion using modern electrophysiologic techniques and ${ }^{31} \mathrm{Phosphorous} \mathrm{magnetic} \mathrm{resonance} \mathrm{spectroscopy,} \mathrm{respectively.}$

The mechanisms of fatigue in healthy muscle will be reviewed, and clinical examples of fatigue in neuromuscular disorders will be presented. Patients with the Chronic Fatigue Syndrome offer a clear example of impaired central activation of motor units (central fatigue) during endurance exercise. The presence of both central factors and peripheral factors will be illustrated in multiple sclerosis patients to highlight the complexity of fatigue in some clinical disorders. Other examples will include the peripheral fatigue of myotonic diseases, McArdle's disease, and mitochondrial myopathies.

This approach permits a more comprehensive analysis of fatigue in a quantitative and non-invasive fashion.

17.

\section{EMG Potentiation - Another Example of Muscle Wisdom}

\section{A.J. McCOMAS (Hamilton, Ontario)}

When a muscle is voluntarily contracted or is stimulated repetitively, the $\mathrm{M}$-wave (muscle compound action potential) frequently becomes larger. In the first second or so this enlargement is probably due to mechanical artifact, but the gradual increase in response which follows has never been explained satisfactorily and is termed "pseudofacilitation". In studies of anaesthetized rats we have shown that the augmentation of the $\mathrm{M}$-wave results from hyperpolarization of the muscle fibres and that the latter, in turn, is a consequence of increased electrogenic $\mathrm{Na}^{+}$pumping. The enhanced pumping is a feature not only of the stimulated fibres but also of those which have hitherto been resting. There are three beneficial effects of the increase in $\mathrm{Na}^{+}$ pumping. First, the rise in interstitial $\left[\mathrm{K}^{+}\right]$is moderated during impulse activity. Second, the stimulated muscle fibres can remain excitable, despite the depolarizing trend brought about by the rise in interstitial $\left[\mathrm{K}^{+}\right]$. Third, the quiescent fibres also remain excitable and can be brought into play if more force is required or if the muscle begins to fatigue. Pharmacological studies indicate that the release of intramuscular norepinephrine is probably one of the stimulants to the $\mathrm{Na}^{+}$pump.

Research supported by: NSERC.

18.

\section{New Insights Into Motor Neuron Firing Behavior and the Generation of Force}

\section{JOE F. JABRE (Boston, U.S.A.)}

In the late 60's Henneman and colleagues described the existence of a size principle which governed the orderly recruitment of motor units. This contribution led to a whole new era in the study of motor control. For once it seemed that the peripheral nervous system actions were not mere effects of central nervous system behavior but rather extensions of, and partners in, the generation of movement.

These inroads to the behavior of firing rates, their alteration in health and in disease and the strategies used by the nervous system to compensate for disturbances in motor control made it essential to begin to develop technologies which allowed for the study of the firing rate behavior of the motor unit potentials and their relationship to the generation of Force.

In this presentation we will review the results of our investigations of the firing rate behavior of upper and lower motor neurons and their role in force generation using the De Luca Precision Decomposition and Macro EMG techniques.

The study was conducted by tracking 5 concurrently active motor unit action potentials (MUAP) in the First Dorsal Interosseous muscle of a 23-year-old subject during a $15^{\prime \prime}$ trapezoid contraction reaching $50 \%$ of Maximum Voluntary Contraction (MVC).

The Frequency Distribution of individual MUAPs firing rates was analyzed and found to be Gaussian. The common behavior of all the active units, the "Common Drive", originates from an upper motor neuron and is also Gaussian in distribution. When the firing rate is corrected for the motor unit's Macro size, this "Rate $\times$ Macro" record is parallel to the Force. It represents the unit's "electrical" twitch at a given firing rate or "Electrotwitch".

Finally, we will describe how to determine a motor unit's type by measuring the electro-mechanical lag between its firing and the Force output. 
19.

\section{Turns-Amplitude Analysis in Botulinum Toxin Treatment of Focal Dystonia}

A. FUGLSANG-FREDERIKSEN, L. ØSTERGAARD, O. SJØ, L. WERDELIN, H. WINKEL and S. PRYTZ (Copenhagen, Denmark)

Botulinum toxin (BT) injected into the dystone muscles is an effective treatment for patients with focal dystonia. An obvious choice for measurement of the degree of hyperactivity of the dystone muscle is the turns-amplitude analysis of the EMG.

In patients with torticollis we sampled the EMG via a concentric needle electrode simultaneously in 4 neck muscles at rest ( 3 sites in each muscle) and at maximal voluntary contraction (MVC) ( 1 site in each muscle). The number of turns/s, the mean amplitude and ratio of turns to mean amplitude were analyzed. Activity of more than 100 turns/s at rest was defined as abnormal.

In the untreated muscles of 44 patients with torticollis an increased turns/s at rest was seen in 12 patients in the sternocleidomastoid muscle contralateral to the involuntary head rotation and the ipsilateral posterior neck muscle, in 13 patients in the contralateral sternocleidomastoid and the ipsi- and contralateral posterior neck muscles and in 7 patients in all 4 muscles. Other combinations were seen less often. At MVC the turns-amplitude analysis indicated that the "healthy" muscles did not activate all their motor units, suggesting that there is a bilateral affection in torticollis patients with overactivity on one side and inhibition on the other side.

In a controlled study of 19 patients with torticollis the turnsamplitude analysis was used as a guidance for BT treatment of muscles with abnormal activity at rest. Botulinum toxin (75 units) was given to 10 subjects and placebo to 9 subjects in a blind randomized manner. Six weeks after the first BT treatment the BT treated muscles showed decreased muscle activity at rest as indicated by reduced turns/s and mean amplitude compared to pretreatment values. Similar changes were seen when comparing BT treatment to placebo.

In another study of 19 patients with torticollis the time course of the effect of BT treatment was studied in the contralateral sternocleidomastoid muscle. After the first BT treatment a second EMG examination for the next BT treatment was performed between 6-30 weeks after the first treatment. The number of turns/s and mean amplitude indicated that the initial reduction in abnormal muscle activity was followed by an increased muscle activity reaching pretreatment values about 30 weeks after the first treatment. An interesting finding was that the ratio of turns to mean amplitude reached values above pretreatment values indicating changes similar to those seen in myopathy probably due to random loss of muscle fibres.

In 15 patients with spasmodic dysphonia the turns-amplitude analysis of the EMG of the vocal muscles was helpful in the diagnosing of the disorder and is a guidance for the BT treatment.

One patient complained of contraction of the trapezius muscles during intonation. Simultaneous EMG of vocal and both trapezious muscles showed activity in both trapezius muscles during intonation, but not during swallowing. BT treatment of the trapezius muscles had a very good effect on the patient. We think, that this patient has an unusual kind of focal dystonia.
In conclusion, quantitative EMG seems useful in the selection of muscles to be treated and in establishing the BT doses.

20.

\section{EMG Measurement of Motor Unit Reorganization and Recruitment Order}

JERZY KOPEĆ (Warsaw, Poland)

In our department, on the basis of more than 20 years' clinical experience in automatic EMG, we have developed a unique computer system for routine clinical examination. The system, called EMG-LAB, provides total integration of IBM PC/AT with any kind of EMG machine. All commercially available methods are based on different matching (template) or decomposition (ADEMG) techniques; these methods are inherently unable to identify and measure total MUAPs (motor unit action potentials) properly, due to the variability of the latter in pathological conditions. In view of this restriction, our method is based on our original concept whereby all EMG signals are processed without loss or distortion. During weak effort all the variably-shaped MUAPs are identified during consecutive recruitment, measured on-line, and then averaged out statistically; the results are presented in histograms. These histograms represent the statistical occurrence of the most significant waveforms among the whole palette of active MUs, together with their variability. This variability of MUAPs, shown in separate histograms, represents respectively duration, amplitude, phases, turns and area; these parameters are strongly related to anatomical reorganization of $\mathrm{MU}$ architecture. Also, the interference pattern (IP) is analyzed in a unique program, in which the sizes and recruitment of MUs are automatically measured and quantitatively assessed. For a global assessment of IP the mean amplitude and mean density are used. The most predominant IP activity, termed "background activity" is generated by low threshold MUs, which in most cases are responsible for compensatory mechanisms and for most of the muscle force. The other clinically important information concerns the activity of the large MUs and is obtained from upper centile amplitude of the record.

Applying these methods in routine clinical examination, we could confirm the traditional criteria for distinguishing normal from pathological, and myopathic from neuropathic diseases, at the level of individual MUAPs; together with the IP, the diagnostic yield was 80 to $100 \%$. We also detected some phenomena not previously emphasized.

Using this fully automatic system for more than two years in routine clinical EMG, we have tried to develop better techniques for evaluating neuromuscular disorders. Therefore, we now include in our analysis the sizes of MUAPs and their recruitment order. To test this concept in a pilot study, four groups of patients with myopathy, Duchenne dystrophy, peripheral neuropathy and motor neuron diseases, respectively, were analyzed. These patient groups were compared with controls and two muscles (biceps brachii and rectus femoris) were examined. First we confirmed that in the control group isometric contraction from weak to maximal contraction was characterized by the recruitment of MUs in an orderly pattern according to the size principle. This basic pattern was used for assessing how recruit- 
ment was distubed in different pathological disorders. In each group of subjects the range of MU sizes, assessed in terms of MUAP amplitudes, was determined from threshold to maximal contractions. For quantification of recruitment order the linear recruitment index was introduced, this being the ratio of the mean MUAP amplitudes during maximal and weak efforts.

We can summarize our data as follows. In myopathic groups all the MU sizes were slightly reduced, either to approximately the same degree, or to a greater extent in the low threshold population. The mean firing rates during maximal contraction were normal or slightly increased; the increased firing rate in background activity indicated that the disease processes affected low threshold MUs more or earlier than the high threshold MUs. Irrespective of the increased variability in size and firing rate, the orderly pattern of recruitment was preserved. A different picture was seen in the group of patients with Duchenne dystrophy, in which the range of MU sizes was diminished, with a characteristic lack of high threshold MUs. At the same time a considerable rearrangement of fiber density within MUs was detected. This change was also reflected in the greatly increased density of the background activity. Evidently the MUs lose their orderly recruitment pattern and linear index. In addition, in both myopathic groups the areas of the MUAPs were reduced on average to $60 \%$.

In the neuropathic groups, the reinnervation process reorganized the surviving MUs, changing their functional effectiveness and thus recruitment order as well as firing rate. In the polyneuropathy and MND patients the increased fibre density within surviving MUs was reflected in the MUAP areas; we found a reduction in the number of functioning MUs and expressed this loss as a percentage. The recruitment pattern was distorted and the linear recruitment pattern was not only reduced but below unity. There was also a large reduction in the range of MU sizes, especially in the MND group. A clear difference between patients with polyneuropathy and MND emerged in MU recruitment order and firing rate. In peripheral neuropathy the surviving MUs, enlarged by collateral reinnervation, were recruited asynchronously during maximal effort, and sometimes gave a "myopathic pattern", with high interference and reduced amplitudes. This finding was probably due to the random reinnervation of muscle fibres by different axons with reduced conduction velocities. In MND patients the surviving MUs showed stronger synchronization and were recruited during maximal effort with high amplitude and low firing rates. In conclusion we suggest that the examination of recruitment order and MUAP parameters can be used as a powerful tool in routine clinical electromyography.

\section{1.}

\section{EMG Interference Pattern Analysis in Infants: A 10 Year Follow-Up Experience}

\section{ARIEH N. GILAI (Jerusalem, Israel)}

Recording and analyzing motor unit action potentials (MUAP's) in neonates, infants and young children is very difficult or even impossible. On the other hand recording and analyzing the EMG interference pattern (IP) is easy, time-saving and provides valid interpretable results.
In 1983 we initiated a program for collecting IP data from infants using specially designed computer hardware and software for data analysis. The main feature of this program was the invention of a threshold detector which enabled us to collect bursts of EMG from the tibialis anterior muscle activated by tickling the sole of the infant's foot. Analysis of these EMG bursts was achieved by measuring and correlating the number of turns and total amplitude in a unit of time (T\&A analysis).

The unique aspect of this work is the fact that we were able to repeat this procedure on the same patient population ten years later, producing results which confirmed our original diagnosis. It is concluded that T\&A analysis of EMG bursts can support a clinical diagnosis by distinguishing normal infants from those with neuromuscular disorders in about $85 \%$ of cases.

\section{2.}

\section{Spectral Modeling in the Analysis of the Interference Pattern}

\section{C.S. PATTICHIS, R. CONSTANTINOU, C.N. SCHIZAS and L.T. MIDDLETON (Nicosia, Cyprus)}

With increasing muscle force the raw EMG signal shows an increase in the number of motor unit action potentials (MUAPs) recruited at increasing firing rates, resulting in the interferencc pattern (IP). Quantitative analysis of the IP was shown to be useful in the diagnosis of neuromuscular disorders and in the past years several computer aided techniques for IP analysis have been proposed including the following: turns amplitude analysis, decomposition methods, and power spectrum analysis. In this work, bispectral analysis, a particular form of higherorder spectra (HOS), is introduced as a new method for analyzing the IP signal. The motivation behind the use of HOS in EMG signal processing is to: detect and characterize existing non linearities in the signal; estimate the phase; and extract information due to deviations from normality.

During the past 30 years several power spectrum estimation techniques were used for the interpretation of the IP signal. These techniques only use second-order statistical information which means that it is assumed that the EMG IP signal is inherently Gaussian. However, the EMG signal is not Gaussian and therefore, conventional spectral techniques often have serious limitations in EMG practice. There is much more information in a signal than conveyed by its autocorellation, or spectrum. Higher-order spectra, which are defined in terms of the higherorder statistics of a signal contain this information. In this presentation HOS will be applied as a new feature extraction process on EMG recorded from normal subjects and patients suffering with neuromuscular disorders. It is anticipated that bispectrum analysis may prove to be a useful tool in the diagnosis of neuromuscular disorders based on EMG.

\section{3.}

\section{New Developments in Nerve Conduction Studies}

W.F. BROWN and T. DOHERTY (Boston, U.S.A.; London, Ontario)

Nerve conduction studies, as commonly practiced, usually limit the assessment of conduction to the distal one-half to one- 
third of the peripheral nervous system although F-responses serve as an indirect means of assessing conduction in the more proximal peripheral nervous system. Recently developed techniques such as percutaneous high voltage electrical stimulation of the spinal roots in the cervical region and ventral roots in the lumbosacral region coupled with collision techniques now make it possible to more directly assess conduction including maximum conduction velocities, temporal dispersion, and conduction block in the proximal one-half to two-thirds as well as the more distal regions of the peripheral nervous system. The application of these techniques often reveals conduction abnormalities in the proximal portions of the peripheral nervous system (PNS) which are not apparent in more conventional distal studies.

As well, the range and frequency distribution of the conduction velocities of single motor axons may be derived using single motor unit action potentials extracted with computer assistance from the F-response. The latter studies have shown that the distribution of conduction velocities for single thenar motor axons is unimodal, and the range of conduction velocities in individual subjects is of the order of $10-20 \%$, and approximately twice that in pooled data. In older subjects (over 65 years of age) the conduction velocities of single thenar motor axons were found to be uniformly slowed with no indication of preferential losses of the more rapidly conducting motor axons.

In healthy median nerves it is sometimes possible to find and stimulate the same motor axon (motor unit) at two or more sites at least $100 \mathrm{~mm}$ apart. This makes direct measurement of the conduction velocity of the motor axon possible. As well, the residual latency of the motor unit can be calculated. For a limited but statistically satisfactory sample of motor units we have found the residual latency to be greater in more rapidly conducting motor axons; a finding which may reflect a more extensive preterminal branching network in these motor units. Determinations of residual latency in longitudinal studies of single motor units may provide a useful means of getting at the terminal innervation patterns of both healthy and disease affected motor units.

As well, recent intraoperative studies of ulnar neuropathies have provided evidence of probably continuous conduction in severely affected nerves. Such evidence rests on finding very slowed conduction (of the order of $5 \mathrm{~m} / \mathrm{s}$ or less) accompanied by very substantial increases in the extracellular currents in the most severely affected region of the nerve.

\section{4.}

\section{Involvement of the Peripheral Nervous System in Chagas' Disease}

\section{ROBERTO E.P. SICA (Buenos Aires, Argentina)}

An electrophysiological and histological study of muscle and peripheral nervous system (PNS) was carried out in humans and in an experimental Chagas' disease (Chd) mouse model. 425 patients with chronic Chd, 34 with acute Chd and 181 mice, experimentally infected with parasites, were investigated. Results were compared with controls. Techniques employed in humans were: conventional EMG, estimated number of motor units, nerve motor and sensory conduction velocities, repetitive nerve stimulation and muscle and sural nerve biopsies. In mice conventional EMG, sciatic nerve conduction time, sciatic nerve action potential amplitude, in vitro mepps and epps were recorded.

About $30 \%$ of patients with chronic Chd showed one or more of the following features: diminished interference pattern, most of the remaining motor units (MUP) had polyphasic shape and increased amplitude; reduced number of functional motor units in the thenar, hypothenar, soleus and/or edb muscles; slow sensory and motor conduction velocities; low sensory action potential amplitude and slight impairment of neuromuscular transmission. Acute Chd patients showed reduced amplitude and poliphasic MUPs, slow motor conduction velocity and impaired neuromuscular transmission. In mice, MUPs duration and amplitude were initially diminished and progressively increased at later stages of the infection, nerve conduction was slow, nerve action potentials were of low amplitude, mepps were of low amplitude and double epps were frequently found. Muscle histology in humans with chronic Chd showed type I and type II grouping. In mice there was found grouped atrophy and angiitis with perivascular infiltrates. Sural nerve samples showed segmental and paranodal demyelination and axonal loss. The same features were observed in mice; also in this model loss of neurones at the dorsal ganglia and at the spinal anterior horn was observed. Vasculitis could be found in every segment of the mouse PNS, infiltrates were mainly composed of T lymphocytes with predominance of Lyt $2+$ or $\mathrm{L} 3 \mathrm{~T} 4+$ subsets according to the strain of parasites employed for infecting the animals.

These findings suggest that the skeletal muscle and the peripheral nervous system may be involved in Chagas' disease.

25.

\section{Reconsideration of the Phenomen of Conduction Block}

\section{W. TROJABORG (New York, U.S.A.)}

$\mathrm{CB}$ can be suspected when there is a discrepancy between the force of a muscle and the amplitude of the compound muscle action potential (CMAP) evoked by distal stimulation of the motor nerve. $\mathrm{CB}$ is diagnosed when three conditions are met: a) the ratio between amplitudes of the evoked CMAP after proximal and distal stimulation of a short nerve segment $(<250 \mathrm{~mm})$ is less than $0.50 ; b)$ the ratio of proximal to distal area under the negative phase of the CMAP is less than 0.50 ; and c) the increase in duration of the negative phase of the CMAP from proximal to distal stimulation is less than $30 \%$. These criteria are rather rigid, but are used to exclude amplitude reduction due to phase cancellation especially when the distally evoked CMAP amplitude is markedly reduced (Rhee et al. 1990' Lange et al. 1992'). The most direct proof is demonstration of slow motor conduction velocity across the damaged segment with absent or delayed F-waves. To ensure the existence of conduction block, the partially blocked CMAP should be compared with the summated muscle action potential produced by maximal volitional effort. The latter is obtained by using an amplitude trigger set at the largest peak of the recruitment pattern during full effort and delayed $40 \%$. Filter and sweep speed settings are the same as those used when evoking the CMAP by electrical stimulation. As measured in that way, the summated muscle 
action potential matches the partially blocked CMAP, confirming the presence of a focal conduction block.

26.

\section{Mononeuritis Multiplex Revisited}

\section{R.E. LOVELACE (New York, U.S.A.)}

Mononeuritis multiplex (MM), originally described in the vasculitis of polyarteritis nodosa ${ }^{1}$ is defined as a neuropathy affecting multiple nerves, usually asymmetrically in anatomical space and at different points in time, giving rise to varying degrees of pathophysiologic and clinical maturity of the lesions. At first, axonal lesions in the collagen vascular diseases were described followed by infective neuritis as in neuropathy of lepromatous leprosy ${ }^{2}$ and the mononeuritic form of diabetic neuropathy, when the development of the disorder had also strong demyelinating aspects.

A revised definition of $\mathrm{MM}$ incorporated these different pathologies giving it syndrome status. ${ }^{3}$ Although neuropraxia is usually thought to describe the temporary disturbance of function seen in nerve pressure lesions, it has been described in the asymmetric vascular occlusive lesions, and we have observed it in the early phases of a sarcoid neuropathy in a patient with rheumatoid arthritis, both eventually developing axonal lesions.

Cases illustrate the use of quantitative methods of following $\mathrm{MM}$ in the predominantly demyelinating and reversible conduction block (CB) syndromes of some paraproteinemias (a macroglobulinemia recently reported by Thomas) and of multifocal CB motor neuropathy simulating Amyotrophic Lateral Sclerosis. MM syndrome necessitates exclusion of HIV related neuropathy.

'Lovelace, R.E. Mononeuritis Multiplex in polyarteritis nodosa. Neurology 1964; 14: 434-442.

${ }^{2}$ Rosenberg. R.N. and Lovelace, R.E. Mononeuritis Multiplex in lepromatous leprosy. Arch Neurol 1968: 19: 310-314.

'Parry, G.J.G. Mononeuritis Multiplex. Muscle \& Nerve 1985; 8: 493-498.

\section{Poster Sessions}

\section{A-1}

\section{The Integrated EMG Lab}

\section{ERIK STÅLBERG, STEFAN STÅLBERG, MATS ÅSTRÖM and PER YTTERBERG (Uppsala, Sweden)}

A description of the present configuration of our neurophysiological laboratory is given.

About 35 PCs are connected to a local network (LANtastic). They are used for booking-billing, administrative statistics, EMG-analysis, reporting, word processing, statistical analysis, desktop publishing and program development. The EMG set up will be shown in some detail. Recording and analysis are made in a PC based system. The results are stored in a server and each physician may review and edit EMGs-CVs in his/her office and produce a report.

The network has modem connection to a satellite EMG laboratory with the same type of $\mathrm{PC}$ based equipment. All $\mathrm{CV}$ and EMG data may be transmitted to our server and the results, although obtained remotely, may be reviewed in the ordinary way after about 90 seconds.

The network has also connections to hospital and university networks, including E-mail service.

Network connection between all PCs in a laboratory has a number of advantages regarding efficiency for daily routines, e.g., for text handling when more than one person is involved, e.g., a physician and a secretary or two or more physicians working on the same manuscript. The connection of EMG systems to the network is of great advantage; review in the office, display of data during rounds of lectures and transmission of data from remote laboratories.

\section{A-2}

\section{Recruitment Pattern of MU in Peripheral and Central Paresis}

Th. VOGT, W.A. NIX, and C. BEER (Mainz, Germany)

Objective: The goal was to see, if the recruitment pattern of single MUs in altered in lesions of the central or peripheral nervous system.

Background: The recruitment order of MUs during voluntary movement is related to their size (size principle), which is fundamental for fine gradation of force. This ability is disturbed in paresis and spasticity. The role of recruitment disturbance in these conditions is not clear.

Method: We studied the recruitment order in normals, stroke patients and patients with peripheral nerve lesions. In the EDC and TA muscle MU size was estimated by macro emg and related to the units recruitment threshold, measured by strain gauge.

Results: In long standing peripheral lesions MUs were enlarged in regard to their macro potential, their recruitment order according to the size principle was preserved by compared to normals, the thresholds were increased.

In stroke patients this order was disturbed as MU size was only weak correlated to their thresholds. Furthermore less units are activated within the measuring range. 